\title{
Sur la similitude des écoulements provoqués par des jets
}

\author{
Jet flow similitude
}

\author{
Jacques Dat * \\ Professeur, INP Toulouse
}

\author{
Christian Fonade* \\ Maitre-Assistant, INP Toulouse
}

\section{Introduction}

Depuis quelques années, les problèmes relatifs à la qualité des eaux sont devenus des préoccupations essentielles des organismes chargés en particulier des réseaux de distribution d'eau potable ou de l'entretien d'aménagements comportant des réserves d'eau naturelles. Il s'agit dans tous les cas de réservoirs de grande capacité, artificiels ou naturels (lacs, ports). Les écoulements qui peuvent y exister sont provoqués, soit par le système d'alimentation et de vidange, soit par la houle, soit éventuellement par des phénomènes de convection dus à une certaine répartition de température ou de masse volumique. Les vitesses induites sont donc toujours très faibles, et il y a en général formation de zones d'eau morte dans lesquelles l'absence de renouvellement peut provoquer l'accumulation d'eaux polluées. Cette pollution peut être traitée par voie chimique ou biologique, mais on peut aussi envisager de provoquer certains écoulements dans ces réservoirs par des phénomènes hydrodynamiques. Dans le cas de réservoirs d'eau potable, ces solutions doivent provoquer le renouvellement constant de toute la masse de liquide contenue dans le réservoir ; lorsqu'il s'agit de lacs ou de ports, ces écoulements doivent permettre une élimination convenable des matériaux en suspension.

Pour les réservoirs d'eau potable, les solutions hydrodynamiques proposées [Réf. 1 et 2] utilisent les jets issus des tuyaux d'alimentation pour provoquer le mélange des diverses couches de fluide et rendre homogène la totalité des eaux du bassin. Ces solutions ne demandent aucune énergie supplémentaire et dépendent seu. lement des caractéristiques des jets et de leur intéraction éventuelle avec les parois ou la surface libre.

* Institut de Mécanique des Fluides de Toulouse, Laboratoire 005 associé au C.N.R.S.
Dans le cadre de la dépollution des lacs ou des ports, on peut aussi utiliser les propriétés des jets : l'énergie nécessaire est relativement faible et la technologie mise en œuvre très simple.

Toutefois, les études sur ouvrages réels sont dans les deux cas très difficiles à réaliser : d'une part l'introduction de traceurs dans les réservoirs d'eau potable est impossible dans la gamme des concentrations nécessaires aux expérimentations ; d'autre part, des mesures in situ dans un lac ou un port ne permettent pas d'obtenir un volume de renseignements suffisant pour déterminer les caractéristiques de l'écoulement obtenu. Les études sont donc réalisées sur modèle réduit. Les rapports d'échelle sont en général assez grands, et de plus lorsqu'il s'agit de lacs ou de ports, les faibles tirants d'eau im. posent souvent d'utiliser une similitude géométrique distordue.

Nous envisagerons donc le problème de la similitude des écoulements de type jets, limités ou non par des parois ou une surface libre. Nous donnerons ensuite l'application de solutions hydrodynamiques à la dépollution d'un lac et d'un port. Les modèles réalisés présentent donc une surface libre et les dimensions verticales sont petites vis-à-vis des dimensions horizontales.

\section{Similitude des jets libres}

Dans tous les cas, les jets formés sur l'ouvrage sont à priori issus de tuyaux à section droite circulaire : ils sont donc à symétrie de révolution. Toutefois, certaines applications peuvent faire intervenir des jets issus d'une fente, dits "jets plans", et nous les envisagerons aussi en considérant des écoulements bidimensionnels.

Les nombres de Reynolds obtenus sur les ouvrages sont toujours de l'ordre $10^{4}$ à $10^{5}$ et correspondent à des jets pleinement turbulents. 
Considérons le schéma classique d'un jet de mur bidimensionnel : il débouche d'une paroi dans un milieu semi-infini rempli du mêtme fluide (Fig. 1). Nous supposons que le profil des vitesses est uniforme dans la section de sortie de la buse.

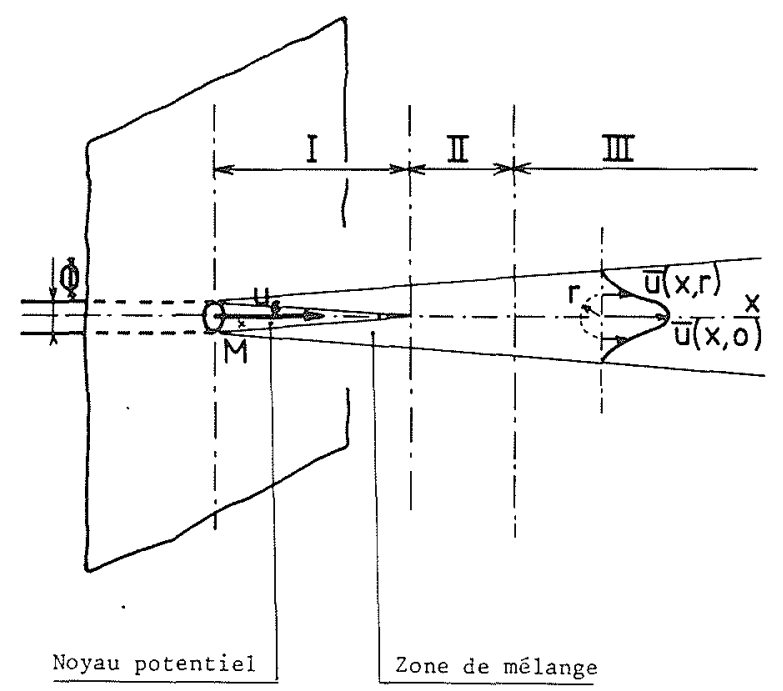

Figure 1

Nous pouvons distinguer trois régions :

- La région I ou "région du noyau potentiel", dont la longueur est de l'ordre de 4 à 5 fois le diamètre $\Phi$ (ou la largeur $\ell$ s'il s'agit d'un jet plan) de la buse et dans laquelle on a toujours des points $M$ où la vitesse longitudinale est égale à la vitesse de sortie $U$ du jet. Ce noyau potentiel est entouré d'une zone de mélange où la vitesse longitudinale passe de $U$ à 0 de façon continue.

- La région II qui est une région de transition.

- La région III ou "région de jet établi" dans laquelle le profil des vitesses moyennes longitudinales $\bar{u}(x, r)$ peut s'exprimer sous la forme :

$$
\bar{u}(x, r)=\bar{u}(x, 0) . f(\eta)
$$

$\eta$ étant une coordonnée adimensionnelle fonction de $x$ et de $r$. Cette région s'étend d'environ $x=15 \Phi$ jusqu'à l'infini aval.

Les solutions analytiques concernant la répartition des vitesses moyennes dans les zones de mélange sont obtenues à partir des équations régissant l'écoulement turbulent d'un fluide incompressible :

$$
\begin{aligned}
& \frac{\partial \bar{u}_{i}}{\partial x_{i}}=0 \\
& \frac{\partial \bar{u}_{i}}{\partial t}+\bar{u}_{j} \frac{\partial \bar{u}_{i}}{\partial x_{j}}=-\frac{1}{\rho} \frac{\partial \bar{p}_{j}}{\partial x_{i}}+\frac{\partial}{\partial x_{j}}\left[\nu \frac{\partial \bar{u}_{i}}{\partial x_{j}}-\overline{u_{i}^{\prime} u_{j}^{\prime}}\right]
\end{aligned}
$$

Le tenseur des contraintes de Reynolds $\left(\overline{u_{i}^{\prime} \overline{u_{j}^{\prime}}}\right)$ n'est pas calculable directement et les solutions analy tiques du système d'équations différentielles précédent sont obtenues en reliant la contrainte $\overline{u_{i}^{\prime}} \vec{u}_{j}^{\prime}$ aux valeurs moyennes des composantes de la vitesse instantanée :

$$
-\overline{u_{i}^{\prime} u_{j}^{\prime}}=\epsilon\left(\frac{\partial u_{i}}{\partial x_{j}}+\frac{\partial u_{j}}{\partial x_{i}}\right)
$$

dans laquelle le paramètre $\epsilon$ est la viscosité cinématique apparente de l'écoulement turbulent. Pour un écoulement bidimensionnel (plan ou à symétrie de révolution), les équations peuvent alors être simplifiées sous la forme d" "équations de la couche limite".

- Equation de continuité :

$$
\frac{\partial}{\partial x}\left(y^{n} \bar{u}\right)+\frac{\partial}{\partial y}\left(y^{n} \bar{v}\right)=0
$$

- Equation dynamique :

$$
\frac{\partial \bar{u}}{\partial t}+\bar{u} \frac{\partial \bar{u}}{\partial x}+\bar{v} \frac{\partial \bar{u}}{\partial y}=-\frac{1}{\rho} \frac{\partial \bar{p}}{\partial x}+\frac{\partial}{y^{n}} \frac{\partial}{\partial y}\left[\epsilon y^{n} \frac{\partial \bar{u}}{\partial y}\right]
$$

le coefficient $n$ étant égal à 0 pour un jet plan et à 1 pour un jet à symétrie de révolution.

$\mathrm{Si}$ nous considérons deux écoulements bidimensionnels (indices 1 et 2) liés par les rapports d'échelles $\lambda$ pour les dimensions, $\alpha$ pour les vitesses, $\beta$ pour les pressions et $\tau$ pour les temps, les équations (4) et (5) donnent les relations de similitude suivantes:

$$
\alpha=\lambda / \tau \quad \beta=\alpha^{2} \rho_{2} / \rho_{1} \quad \epsilon_{2} / \epsilon_{1}=\alpha \lambda
$$

Les deux premières relations sont classiques et permettent d'obtenir les rapports des vitesses et des pressions. La dernière relation lie le rapport des viscosités cinématiques apparentes aux rapports d'échelle des longueurs et des vitesses. Ce paramètre $\epsilon$, qui est fonction du point considéré, peut être, en première approximation exprimé en fonction des caractéristiques de vitesse moyenne en ce point. Prenons par exemple l'hypothèse telle que $\epsilon$ soit constant dans une section transversale du jet et qu'il s'écrive sous la forme :

$$
\epsilon=\chi b \bar{u}_{\max }
$$

expression dans laquelle $b$ est une dimension caractéristique de la zone de mélange, $\bar{u}_{\max }$ la valeur maximum de $\bar{u}$ dans la section considérée et $\chi$ un coefficient de proportionnalité.

On a donc : $\epsilon_{2} / \epsilon_{1}=\left(\chi_{2} / \chi_{1}\right) . \lambda \alpha$

La relation (6) sera donc vérifiée si $\left(\chi_{2} / \chi_{1}\right)$ est égal à 1 . Or, l'expérience montre que le coefficient expérimental $\chi$ ne dépend que du type de jet envisagé (plan ou à symétrie de révolution) et est indépendant de l'échelle géométrique.

Par conséquent les relations (6) n'imposent aucune condition et la similitude des vitesses moyennes entre deux jets bidimensionnels libres d'un fluide incompressible est toujours possible.

En conséquence, le profil longitudinal de vitesse sur l'axe $\bar{u}(x, 0)$ et les profils transversaux affines du type $\bar{u}(x, y) / \bar{u}(x, 0)=f(\eta)$ sont conservés lorsqu'on applique la similitude. Comme le paramètre $\eta$ est proportionnel au rapport $(y / x)$, il y aura conservation de la carte des vitesses dans tout l'écoulement, c'est à dire qu'on a la relation :

$$
\frac{\bar{u}_{2}\left(x_{2}, y_{2}\right) / U_{2}}{\bar{u}_{1}\left(x_{1}, y_{1}\right) / U_{1}}=\frac{\bar{u}_{2}\left(\lambda x_{1}, \lambda y_{1}\right) / U_{2}}{\bar{u}_{1}\left(x_{1}, y_{1}\right) / U_{1}}=1
$$


Il apparaît par conséquent que pour un écoulement du type jet libre, la similitude est toujours possible : ayant choisi le rapport $\lambda$, on peut encore choisir le rapport des vitesses $\alpha=\frac{\bar{u}_{2}(\lambda x, \lambda y)}{\bar{u}_{1}(x, y)}=\frac{U_{2}}{U_{1}}$. Si on conserve alors le même fluide, les échelles des temps et des pressions seront :

$$
\tau=\lambda / \alpha \quad \text { et } \quad \beta=\alpha^{2}
$$

et l'échelle des débits :

$$
Q_{2} / Q_{1}=\alpha \lambda^{2}
$$

Ces relations sont bien vérifiées expérimentalement pour les valeurs moyennes de la vitesse, c'est-à-dire les caractéristiques statiques.

En ce qui concerne les phénomènes dynamiques, on constate une certaine instabilité des limites des zones de mélange ; celle-ci se traduit pour un jet plan et des nombres de Reynolds de quelques milliers, par l'existence de rues de tourbillons symétriques ou alternés dont la fréquence proportionnelle au rapport $(U / \ell)$, vérifie donc l'échelle des temps définie par les relations de similitude. Quant à la turbulence elle-même, c'est un phénomène aléatoire qui ne peut donc faire intervenir d'échelle des temps. Toutefois, il est intéressant de noter que ses effets se stabilisent à partir d'un nombre de Reynolds de quelques milliers. Compte tenu des valeurs de $\mathcal{R}$ obtenues sur les ouvrages, celà permet de ne pas imposer une similitude de Reynolds mais de s'assurer uniquement que le nombre de Reynolds obtenu sur le modèle correspond à un écoulement turbulent bien établi.

Les nombreuses études effectuées sur des jets libres plans ou à symétrie de révolution, de dimensions très différentes ou utilisant divers fluides, montrent que les relations de similitude (7), (8) et (9) sont bien vérifiées. Les résultats obtenus dans le cadre de réservoirs d'eau potable [réf. 1] montrent qu'il en est de même lorsque les parois ou la surface libre limitant l'écoulement sont suffisamment éloignées du jet pour ne pas provoquer de modification notable des premières régions du jet qui sont les zones les plus actives pour l'écoulement global. Les essais de vérification ont été réalisés dans deux bassins parallélépipédiques (Fig. 2) avec un rapport d'échelle géométrique $\lambda$ de $1 / 10$. La figure 3 donne la variation du coefficient $\Delta C$ lié à la concentration en

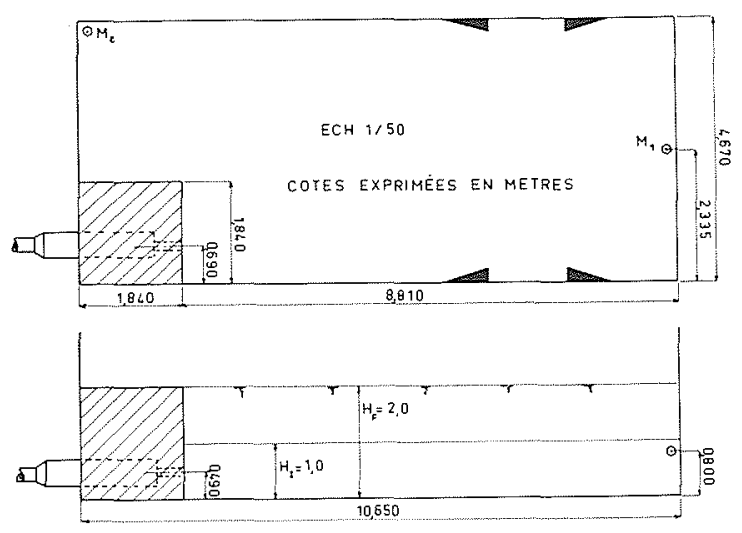

Figure 2

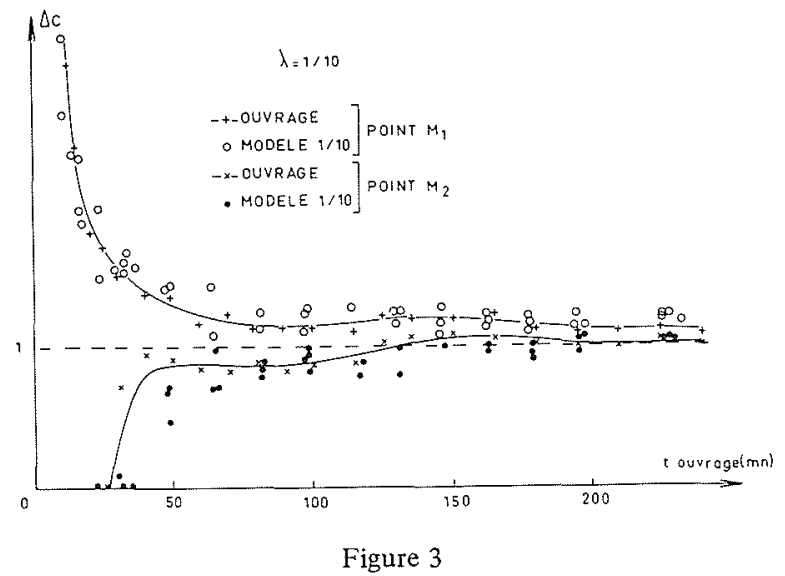

bichromate de soude de l'eau aux points $M_{1}$ et $M_{2}$. On constate une bonne concordance des résultats malgré la position très défavorable des points de mesure par rapport à la géométrie du bassin, et donc de l'écoulement.

\section{Similitude des jets confinés}

La présence de parois est impérative lorsqu'il s'agit d'écoulements de liquides et il est intéressant d'analyser leur influence sur le comportement des jets. Les caractéristiques moyennes des jets libres que nous avons rappelées dans le paragraphe précédent, montrent que c est dans les deux régions I et II (Fig. 1) que le jet est le plus sensible à une action extérieure. Les gradients de vitesse moyenne y sont importants et une perturbation extérieure de faible amplitude peut déséquilibrer le jet et modifier de façon notable la géométrie de l'écoulement aval.

La présence de parois ou d'écoulements secondaires dans ces zones devient donc un des paramètres important. Un certain nombre d'études ont été réalisées dans ce cadre : nous pouvons citer en particulier, l'étude de l'écoulement d'un jet bidimensionnel entre parois par M. Curtet [Réf. 3].

Toutefois, les problèmes qui nous préoccupent ici font intervenir, d'une part des parois qui sont situées loin de la buse et ne peuvent donc influencer directement les zones de mélange des régions initiales du jet, et d'autre part un radier et une surface libre placés à proximité de l'axe du jet. Nous n'envisageons donc ici que l'influence de ce dernier type de confinement sur l'application pratique des lois de similitude.

En ce qui concerne le comportement statique du jet, déterminé essentiellement par les conditions existant près de la buse, seuls interviennent le radier et la surface libre. Considérons par exemple un jet de mur de diamètre $\Phi$ débouchant horizontalement à proximité d'une surface libre (Fig. 4) tel que le rapport $\Phi / z_{0}$ ne soit pas trop petit. On peut trouver une solution analytique à condition toutefois de s'affranchir de la non-linéarité des équations dynamiques de la couche limite. Ainsi, moyennant certaines hypothèses et en particulier celle d'un profil affine de quantités de mouvement, on obtient un système différentiel linéaire qui autorise 


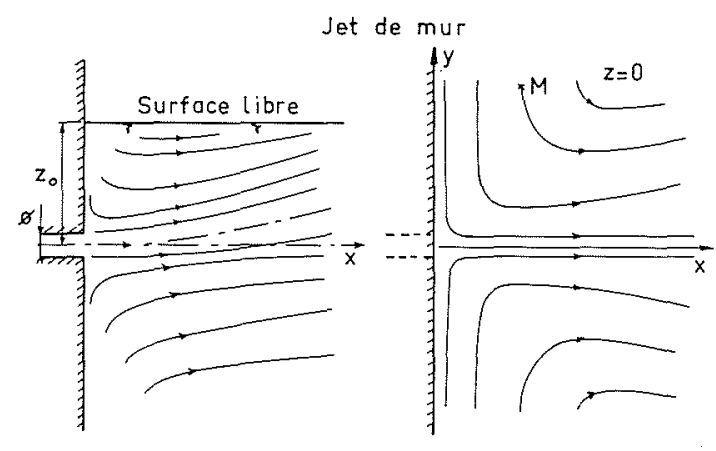

Figure 4

l'application du principe de superposition. En utilisant alors la méthode des images, on prend en compte l'existence d'une surface libre et on obtient la répartition des vitesses dans le demi espace $x>0$ [Réf.4] sous la forme :

$$
\begin{aligned}
& \frac{\overline{u^{2}}}{U^{2}}=\alpha {\left[\operatorname{Exp}\left[-\frac{4 \alpha}{\Phi^{2}}\left(y^{2}+\left(z-z_{0}\right)^{2}\right)\right]\right.} \\
&+\left.\operatorname{Exp}\left[-\frac{4 \alpha}{\Phi^{2}}\left(y^{2}+\left(z+z_{0}\right)^{2}\right)\right]\right] \\
& \alpha=1-\operatorname{Exp}\left[-\frac{\Phi^{2}}{4 c^{2} x^{2}}\right]
\end{aligned}
$$

avec : $x$ coordonnée selon l'axe de la buse

$y$ coordonnée normale à $x$ dans un plan horizontal

$z$ coordonnée verticale descendante $(z=0$ à la surface libre)

$z_{0}$ cote de l'axe de la buse

$c$ constante déterminée expérimentalement

Remarquons que ce résultat suppose une surface libre indéformable : la buse étant toujours noyée, il n'y a jamais de phénomène de ressaut et cette hypothèse est parfaitement justifiée dans les modèles hydrauliques qui nous concernent.

La présence de la surface libre provoque donc un infléchissement de l'axe du jet vers cette surface et à partir d'une distance $x$ d'environ trente fois le diamètre, les profils de vitesse s'écartent notablement de ceux d'un jet libre.

Nous constatons cependant que si l'on applique une similitude géométrique, la relation (10) reste inchangée et la répartition des profils $u^{2} / U^{2}$ est donc conservée. Compte tenu de la relation:

$$
\overline{u^{2}}=\overline{u^{2}}+\overline{u^{\prime 2}}
$$

et de la conservation de la répartition de l'intensité fluctuante $\left(\overline{u^{\prime 2}}\right)^{1 / 2} / U$ pour un écoulement turbulent établi, les profils de vitesse moyenne $\bar{u}(x, y, z) / U$ sont invariants quelle que soit l'échelle géométrique. La présence d'une surface libre ne modifie donc pas les relations de similitude. Il en serait de même de l'influence du radier. Toutefois et compte tenu de la réduction des dimensions, les phénomènes de tension superficielle ou de rugosité peuvent être difficiles à cerner et il faut donc être prudent au niveau de l'interprétation des écoulements près de tels obstacles. Il en est par exemple ainsi pour un seuil déversant pour lequel on doit avoir une charge minimum compatible avec le phénomène de capillarité et donc de tension superficielle.

En ce qui concerne les phénomènes dynamiques, la présence de parois, même situées assez loin du jet, peut provoquer des instabilités auto-entretenues. Les oscillations peuvent être dues, soit à des propagations d'onde, soit à des courants de recirculation. Dans ce dernier cas, les vitesses induites dans le fluide étant liées à la quantité de mouvement initiale du jet, ces contre-réactions agissent, soit par pression s'il y a des points d'arrêt, soit par quantité de mouvement si des écoulements de retour existent. Elles suivent alors les lois de similitude obtenues pour un jet libre.

Il n'en est pas de même si l'entretien des oscillations est provoqué par des phênomènes d'onde : le rapport d'échelle des temps sera donné par le rapport :

$$
\tau_{c}=\lambda\left(c_{2} / c_{1}\right)
$$

$c$ étant la vitesse de propagation de l'onde dans le fluide considéré. La surélévation de l'intumescence est toujours faible dans les systèmes que nous envisageons et le rapport $\left(c_{2} / c_{1}\right)$ est donc égal à $\lambda^{1 / 2}$.

Pour n'avoir qu'une seule échelle des temps, il faut donc que $\tau=\tau_{c}$, soit :

$$
\lambda / \alpha=\lambda\left(c_{2} / c_{1}\right)
$$

Le paramètre $\alpha$ étant indéterminé pour l'instant, on vérifie cette relation en prenant : $\alpha=\lambda^{1 / 2}$.

Dans ce dernier cas on obtient les lois de similitude de Reech-Froude applicables à un système présentant une surface libre.

La conservation d'une seule échelle des temps pour les phénomènes statiques et dynamiques impose donc une condition sur l'échelle des vitesses. Ce dernier paramètre étant encore indéterminé, on peut donc réaliser la similitude d'écoulements turbulents du type jet en milieu confiné. Dans le cas par exemple d'un écoulement présentant une surface libre, on a les relations de similitude de Froude :

$$
\begin{array}{ll}
\text { vitesses }: & \alpha=\lambda^{1 / 2} \\
\text { temps }: \tau=\lambda^{1 / 2} & \\
\text { débits }: Q_{2} / Q_{1}=\lambda^{5 / 2}
\end{array}
$$

\section{Cas d'une similitude géométrique distordue}

L'utilisation d'une similitude géométrique distordue est très souvent imposée par les grandes différences existant entre les dimensions horizontales et verticales de l'ouvrage. Ce sera par exemple ie cas dans l'étude du modèle d'un port pour lequel on est amené à prendre un rapport d'échelle horizontale $\lambda_{H}$ et un rapport d'échelle verticale $\lambda_{V}$.

On peut alors tout d'abord admettre la conservation relative des sections de passage : les sections des buses formant les jets ouvrage et modèle sont alors dans le rapport $\left(\lambda_{H} \cdot \lambda_{V}\right)$. Si on représente alors un jet ouvrage de section droite circulaire (diamètre $\Phi$ ) par un jet modele de section rectangulaire (largeur $\ell$, hauteur $h$ ), on a la première relation: 


$$
\ell . h=\left(\lambda_{H} \cdot \lambda_{V}\right)\left(\pi \Phi^{2} / 4\right)
$$

Le rapport de distorsion $\delta$ se retrouvant dans les dimensions de la buse, on a de plus :

$$
\delta=\lambda_{V} / \lambda_{H}=h / \ell
$$

Dans ces conditions la relation (10) relative à un jet circulaire en présence d'une surface libre, ne permet pas d'obtenir un critère de similitude simple pour le rapport $\left(u^{2} / U^{2}\right)$ des quantités de mouvement. Il y a en effet indétermination sur la longueur de référence que l'on doit associer à $\Phi$ dans le cas d'un jet rectangulaire.

Nous avons donc réalisé des mesures de vitesse dans le demi espace $x>0$ relatif à l'écoulement créé par un jet issu d'une buse rectangulaire de rapport de distorsion égal à $3(\ell=3 \mathrm{~mm}, h=9 \mathrm{~mm})$. La maquette expérimentale comporte un système de déplacement du porte sonde dans les trois directions $x, y, z$, la position effective de la sonde étant mesurée par trois potentiomètres liés aux vis assurant les déplacements. La chaîne de mesure anémométrique comporte en particulier un anémomètre à fil chaud "à température constante" associé à un linéarisateur ; le signal obtenu (proportionnel à la vitesse) peut être enregistré ou visualisé sur un oscillographe cathodique.

A partir des profils expérimentaux $\bar{u}(x, y, 0)$ et $\bar{u}(x, 0, z)$ (Fig. 5) nous pouvons obtenir les caractéristiques statiques d'un tel jet. On constate tout d'abord que le noyau potentiel est plus court que celui d'un jet plan : l'épanouissement possible du jet dans les directions $y$ et $z$ est compensé par une décroissance initiale plus rapide de la vitesse sur l'axe. Dans la région de jet établi, les profils $\bar{u}(x, y, 0)$ peuvent être représentés par un profil affine : le pôle du jet se situe pour cette buse en $x=0$.

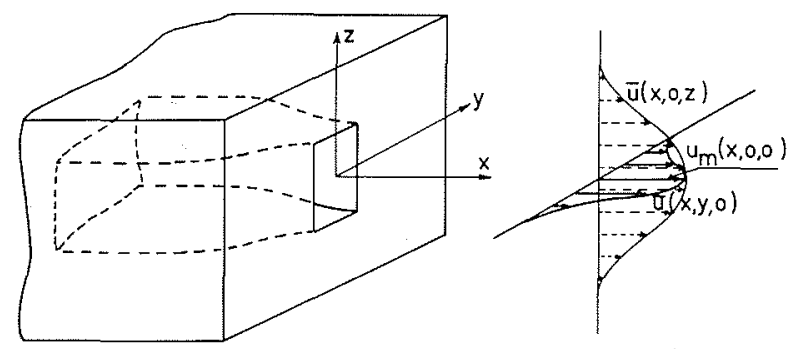

Figure 5

La figure 6 donne les résultats expérimentaux $\bar{u}_{m}(x, 0,0)$ de la vitesse maximum dans la section $x$ considérée, et les courbes correspondant respectivement à un jet plan de largeur $\ell$ et à un jet à symétrie axiale de diamètre $\phi,(\phi$ étant défini par l'égalité des sections : $\left.\pi \phi^{2} / 4=\ell h\right)$. On constate que pour les petites valeurs de $x$, le profil $\bar{u}_{m}(x, 0,0)$ correspond plutôt à celui d'un jet plan tandis que pour les grandes valeurs de $x(x>12 \phi)$ cette caractéristique a l'allure en $(k / x)$ de la variation de la vitesse longitudinale sur l'axe d'un jet circulaire. D'autre part la figure 7 montre que jusqu'à cette distance, le jet s'épanouit de telle façon que l'on puisse considérer que la longueur caractéristique est $\ell$ pour les profils $\bar{u}(x, y, o)$ et $h$ pour les profils $u(x, o, z)$.

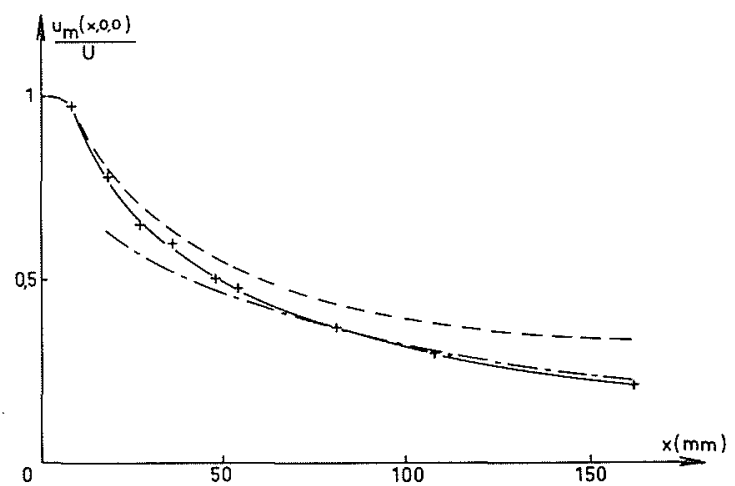

Figure 6

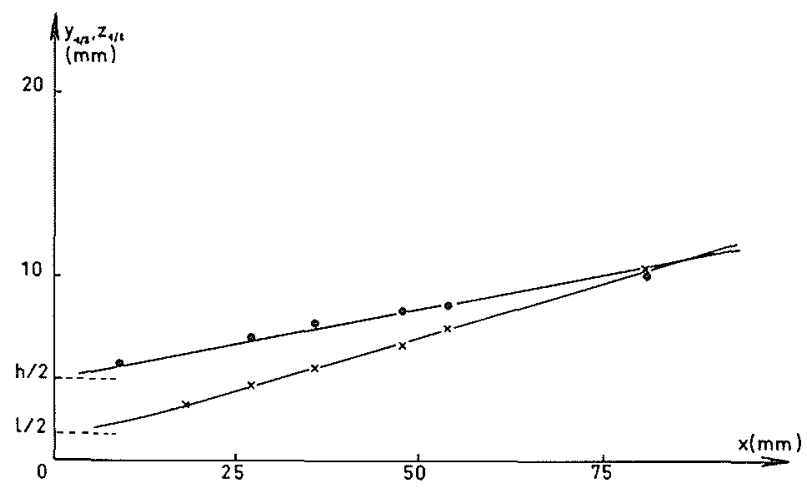

Figure 7

Considérons alors la représentation d'un jet circulaire pour une similitude géométrique distordue et supposons que la relation (10) rende compte de la distribution des quantités de mouvement. Elle s'écrit, pour l'ouvrage :

$$
\begin{aligned}
\frac{\overline{u_{1}^{2}}}{U_{1}^{2}}=\alpha_{1}\left[\operatorname{Exp}\left[-\frac{4 \alpha_{1}}{\Phi^{2}}\left(y_{1}^{2}+\left(z_{1}-z_{01}\right)^{2}\right)\right]+\right. \\
\left.+\operatorname{Exp}\left[-\frac{4 \alpha_{1}}{\Phi^{2}}\left(y_{1}^{2}+\left(z_{1}+z_{01}\right)^{2}\right)\right]\right] \\
\alpha_{1}=1-\operatorname{Exp}\left[-\frac{\Phi^{2}}{4 c^{2} x_{1}^{2}}\right]
\end{aligned}
$$

Pour le modèle, nous considérons que dans la région proche de la buse ( $x$ petit), le jet rectangulaire se comporte comme un jet de diamètre $\ell$ pour les profils de vitesse dans des plans horizontaux $(z=$ cte) et comme un jet de diamètre $h$ pour les profils de vitesse dans les plans verticaux $(y=$ cte). Cette hypothèse permet de rendre compte des dimensions relatives des zones de mélange s'épanouissant à l'aval de la buse. La répartition des quantités de mouvement s'écrit alors :

$$
\begin{aligned}
& \frac{\overline{u_{2}^{2}}}{U_{2}^{2}}=\alpha_{2}[\operatorname{Exp}[\left.-4 \alpha^{2}\left(\frac{y_{2}^{2}}{\ell^{2}}+\frac{\left(z_{2}-z_{02}\right)^{2}}{h^{2}}\right)\right]+ \\
&\left.+\operatorname{Exp}\left[-4 \alpha^{2}\left(\frac{y_{2}^{2}}{\ell^{2}}+\frac{\left(z_{2}+z_{02}\right)^{2}}{h^{2}}\right)\right]\right] \\
& \alpha_{2}=1-\operatorname{Exp}\left(-\ell^{2} / 4 c^{2} x_{2}^{2}\right)
\end{aligned}
$$


Les répartitions $\left(\overline{u_{1}^{2}} / U_{1}^{2}\right)$ et $\left(\overline{u_{2}^{2}} / U_{2}^{2}\right)$ sont les mêmes si les relations (14) et (15) sont identiques, c'est-à-dire si on a :

$$
\frac{x_{2}}{x_{1}}=\frac{y_{2}}{y_{1}}=\frac{\ell}{\Phi} \quad \text { et } \quad \frac{z_{2}}{z_{1}}=\frac{z_{02}}{z_{01}}=\frac{h}{\Phi}
$$

Les rapports d'échelle horizontale et verticale étant respectivement $\lambda_{H}$ et $\lambda_{V}$ on devrait donc avoir :

$$
\frac{\ell}{\Phi}=\lambda_{H} \quad \text { et } \quad \frac{h}{\Phi}=\lambda_{V}
$$

Or les sections étant conservées dans le rapport $\left(\lambda_{H} \lambda_{V}\right)$, la relation (12) permet d'obtenir compte tenu du rapport de distorsion :

$$
\frac{\ell}{\Phi}=\sqrt{\frac{\pi}{4}} \lambda_{H} \quad \text { et } \quad \frac{h}{\Phi}=\sqrt{\frac{\pi}{4}} \lambda_{V}
$$

On obtient donc un écart de l'ordre de $10 \%$ sur les échelles géométriques.

$\mathrm{Si}$ on se place dans la région de jet établi, nous avons vu qu'effectivement le jet rectangulaire est assimilable ’à un jet de section circulaire pour lequel nous prenons un diamètre $\phi$ qui donne égalité des sections donc des débits. La répartition des quantités de mouvement sur le modèle est alors donnée par la relation:

$$
\begin{aligned}
\frac{\overline{u_{2}^{2}}}{U_{2}^{2}}=\alpha_{2}[\operatorname{Exp}[ & \left.-\frac{4 \alpha_{2}}{\phi^{2}}\left(y_{2}^{2}+\left(z_{2}-z_{02}\right)^{2}\right)\right]+ \\
& \left.+\operatorname{Exp}\left[-\frac{4 \alpha_{2}}{\phi^{2}}\left(y_{2}^{2}+\left(z_{2}+z_{02}\right)^{2}\right)\right]\right]
\end{aligned}
$$

$\alpha_{2}=1-\operatorname{Exp}\left[-\phi^{2} / 4 c^{2} x_{2}^{2}\right]$

On a donc même répartition des quantités de mouvement si on a la relation :

$$
\frac{x_{2}}{x_{1}}=\frac{y_{2}}{y_{1}}=\frac{z_{2}}{z_{1}}=\frac{z_{02}}{z_{01}}=\frac{\phi}{\Phi}
$$

Le rapport $(\phi / \Phi)$, qui est égal à la racine carrée du rapport des sections $\left(\lambda_{H} \lambda_{V}\right)$ doit d'une part être égal à $\lambda_{H}$ dans un plan horizontal et à $\lambda_{V}$ dans un plan vertical. Cela n'est donc pas réalisable, et on a un écart d'autant plus important que le rapport de distorsion est grand.

Il apparaît ainsi que lorsque les rapports d'échelles horizontale et verticale sont différents, la similitude géométrique n'est pas vérifiée au niveau de la répartition des quantités de mouvement et donc des vitesses. Toutefois, l'écart que l'on peut espérer est le plus faible dans la première zone de l'écoulement, c'est-à-dire dans la région la plus sensible du jet et celle qui détermine effectivement l'écoulement global. Les régions situées à l'aval correspondent à des vitesses et des gradients de vitesse beaucoup plus faibles : la condition d'écoulement turbulent établi devient alors aléatoire sur le modèle et cette approximation s'ajoute aux influences dues aux phénomènes parasites, tels que la rugosité, qui peuvent alors jouer un rôle essentiel. L'expérience montre toutefois que des modifications notables de géométrie dans les régions de faibles vitesses n'agissent que très peu au niveau des jets où l'énergie de ces derniers est concentrée dans un espace restreint.

\section{Application à la dépollution de bassins}

Outre leur application dans les réservoirs d'eau potable, des solutions basées sur des écoulements provoqués par des jets peuvent être envisagées pour l'élimination des matériaux polluants flottants à la surface de lacs ou de ports. Nous citons ici les études que nous avons réalisées dans le cadre des aménagements du lac "Strofylia" de l'île Skiathos et du port de Zeas du Pirée. Les solutions proposées représentent deux types de fonctionnement possible, l'un intermittent, l'autre continu, chacun étant adapté aux problèmes particuliers auxquels il s'applique.

\section{Aménagement du lac Strofylia}

Il s'agit d'un lac d'environ $500 \mathrm{~m}$ de long et $200 \mathrm{~m}$ de large relié à la mer par un chenal. Vu le contexte touristique de cet ensemble, il était nécessaire d'éliminer les matériaux en suspension provenant de la dégradation du radier lui-même et que l'on pouvait par conséquent rejeter à la mer. La figure 8 montre le modèle de ce lac, réalisé selon les échelles $\lambda_{H}=1 / 300$ et $\lambda_{V}=1 / 50$.

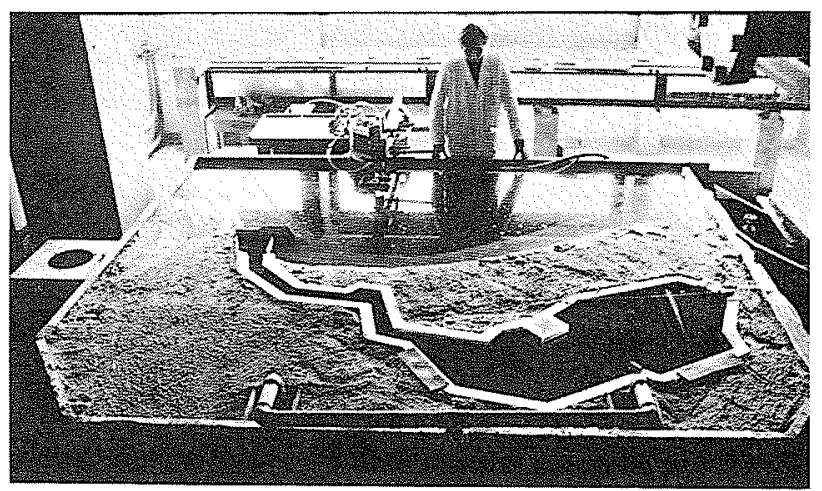

Figure 8

La navigation éventuelle ne devant se faire que durant la journée, nous avons étudié une solution intermittente permettant de réduire l'énergie nécessaire et d'imposer des vitesses assez grandes durant la nuit [Réf. 5]. Avec une puissance de l'ordre de $30 \mathrm{KW}$ (non compte tenu des pertes de charge dans la canalisation d'amenée), l'écoulement atteint sa géométrie stable au bout de 4 heures (Fig. 9) ; l'écoulement se maintient pendant une durée égale lorsque le jet est supprimé.

On constate sur la figure 9 l'existence de plusieurs rouleaux de recirculation permettant de mettre en mouvement la totalité des eaux du lac. Les frontières entre ces rouleaux étant essentiellement et localement instables, un certain échange existe entre ces différentes masses de fluide. Cela permet, par l'intermédiaire de la mise en pression de la zone du point d'arrêt situé le plus 
à l'aval, d'évacuer de façon continue les matériaux flottants vers le chenal et la mer. Des essais dans le temps ont montré que des particules situées dans la zone la plus défavorable du lac (renfoncement situé à droite de la figure 8) mettent deux heures pour atteindre l'entrée du chenal.

Le fonctionnement intermittent permettant d'alimenter le jet à des vitesses assez grandes ( 9 à $10 \mathrm{~m} / \mathrm{s}$ ), la puissance installée est assez importante. En contrepartie un fonctionnement de quelques heures par jour de la station de pompage permet de maintenir un mouvement permanent dans toutes les zones du lac et une évacuation continue vers la mer.

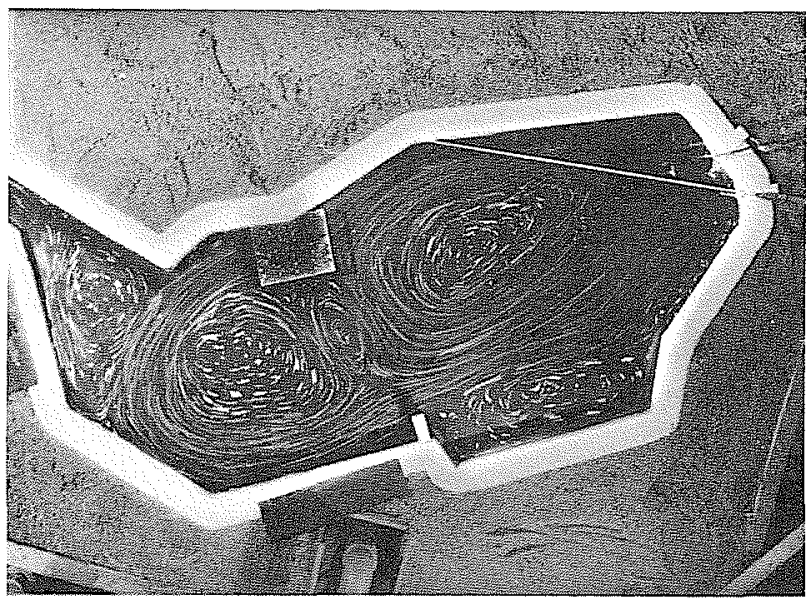

Figure 9

\section{Aménagement du port de Zéas}

Il s'agit là d'un port constitué de deux bassins dans lesquels le mouvement moyen de l'eau est pratiquement nul. Il y règne une pollution importante provenant des habitations envirunnantes et des bateaux. D'autre part, la navigation $y$ étant permanente, les vitesses induites doivent être compatibles avec les possibilités de mouvement des petites embarcations. Nous avons donc étudié une solution continue.

Réalisé selon les échelles $\lambda_{H}=1 / 100$ et $\lambda_{V}=1 / 50$, le modèle couvre environ $150 \mathrm{~m}^{2}$ (Fig. 10) et présente des tirants d'eau variant entre 4 et $40 \mathrm{~cm}$. Il comporte deux bassins reliés par un chenal, le bassin le plus éloigné de la mer étant le plus pollué et le moins profond (fig. 11). La pollution provenant de causes extérieures au milieu naturel, nous avons choisi une solution permettant l'élimination des corps flottants. Huit jets à faible énergie sont répartis sur le pourtour du premier bassin : ils provoquent d'une part le brassage de la totalité des eaux et d'autre part un léger écoulement moyen de rotation dans ce bassin. La présence d'un jet situé à l'entrée du chenal provoque alors l'entraînement continu de matériaux vers l'aval (Fig. 12). Ce dernier jet est maintenu dans la direction du quai par effet d'attachement : le jet pariétal ainsi formé ne provoque pas de vitesses inadmissibles dans l'axe du chenal. Un système de déversoirs latéraux implantés le long de ce jet et sur la partie aval du chenal permet l'élimination des maté-

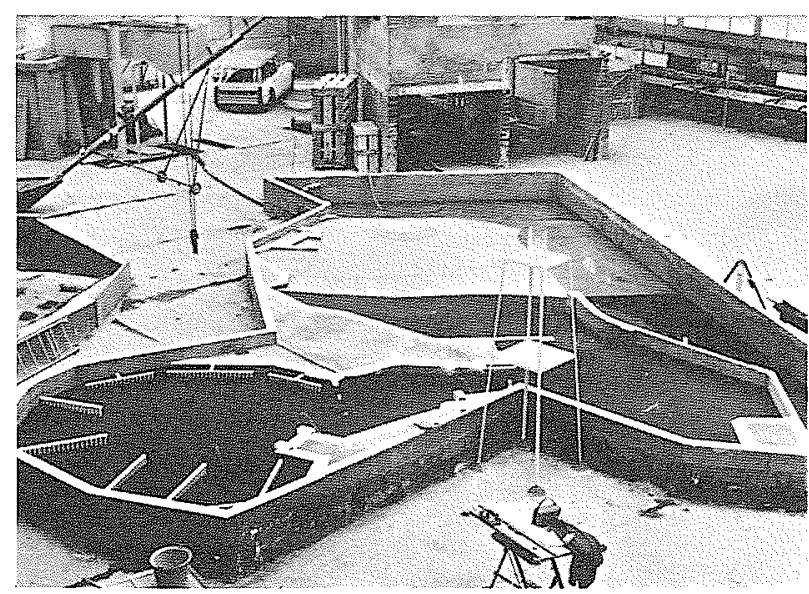

Figure 10

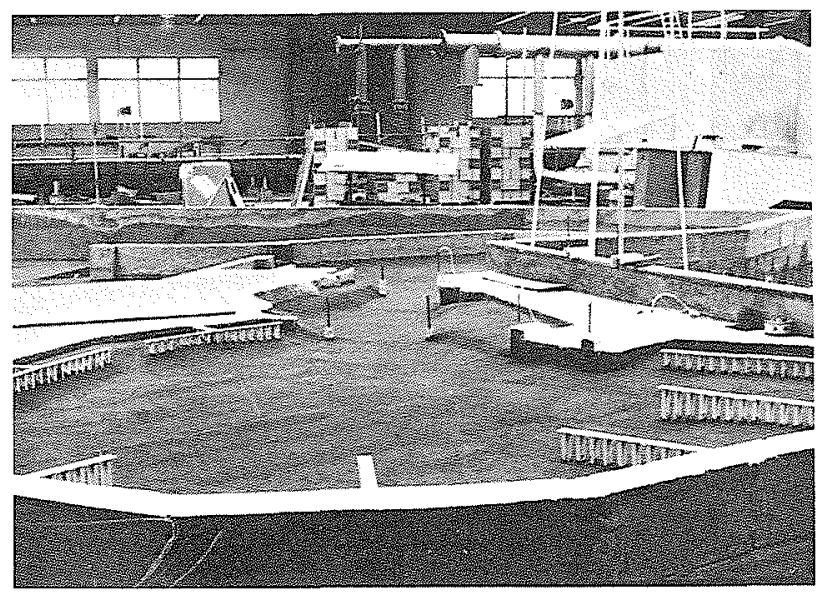

Figure 11

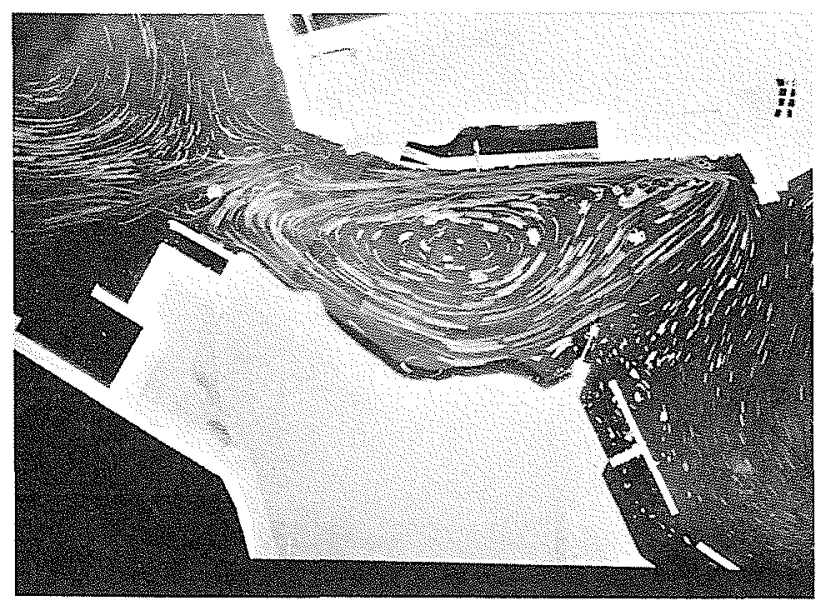

Figure 12

riaux flottants (Fig. 13). Un traitement éventuel des eaux recueillies peut permettre leur utilisation pour l'alimentation des jets dont le débit total ouvrage est de l'ordre de $0,7 \mathrm{~m}^{3} / \mathrm{s}$. Compte tenu des faibles valeurs de la vitesse à la sortie des jets ( 3 à $4 \mathrm{~m} / \mathrm{s}$ ), la puissance nécessaire (non compte tenu des pertes de charge) est de l'ordre de $2,5 \mathrm{KW}$. 


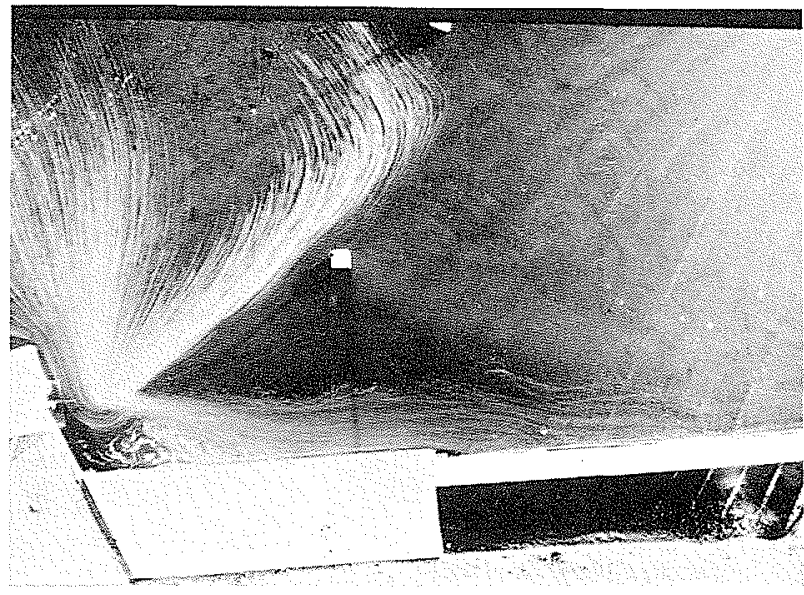

Figure 13

\section{Conclusions}

Les propriétés de mélange et d'entraînement d'un jêt turbulent, la stabilité des écoulements provoqués ou par contre leur sensibilité éventuelle à une perturbation extérieure permettent l'utilisation des jets dans des domaines aussi divers que la réalisation d'automatismes à fluide miniaturisés ou la mise en mouvement de grands volumes de liquides.

Les relations de similitude applicables à de tels écoulements, confinés ou non, montrent qu'il est possible de réaliser correctement les études sur modèle. La condition d'écoulement turbulent pleinement établi dans les premières zones du jet conduit à une similitude du type Reech-Froude qui, compte tenu des problèmes posés, donne des résultats convenables, même dans le cadre d'une similitude distordue. Cependant, un grand soin doit être apporté à la réalisation du modèle (radier, obstacles) et l'interprétation des phénomènes dynamiques pouvant apparaître ou non sur le modèle doit être faite avec beaucoup de discernement.

L'étude sur modèle fournit alors des enseignements concrets sur les solutions qui peuvent résoudre des problèmes tels que ceux que nous avons évoqués.

\section{Remerciements}

Les travaux qui font l'objet de cette publication ont été effectués à l'Institut de Mécanique des Fluides de Toulouse et ont été financés par la Société Tetracktys (Athènes). Nous tenons à remercier particulièrement Monsieur Gérard Claria, technicien à l'I.M.F.T. pour son active participation à cette étude.

\section{Références}

[1] BOULE P., CASTEX L, FONADE C - Le mélange des eaux à l'intérieur d'un réservoir circulaire. Techniques et Sciences Municipales - Juin 1973.

[2] COOLEY P., STEPHEN L., HARRIS M.A. - The prevention of stratification in reservoirs. Journal of the Institution of water engineers, vol. $8, \mathrm{n}^{\circ} 7$, novembre 1954.

[3] CURTET - Sur l'écoulement d'un jet entre parois P.S.T. $\mathrm{n}^{\circ} 359(1960)$

[4] MAXWELL C., PAZWASH H. - Axisymmetric shallow submerged turbulent jets. Journal of the Hydraulic division Avril 1973.

[5] DAT J., FONADE C. - Aménagement du Lac Strofylia de l'ile Skiathos. I.M.F.T. rapport $n^{\circ} 289-1$.

[6] DAT J., FONADE C. - Aménagement du port de Zeas (Grèce). I.M.F.T. rapport n ${ }^{\circ} 310-2$. 